Nevertheless, the high quality of current observations, particularly seismic data, dictates that the best possible models should be used. The equation of state used in the OPAL opacity tables is based on many-body quantum statistical methods (Rogers, $1994 ; 1986 ; 1981$ ) and is suitable for the modeling of seismic data. Extensive tables of the OPAL equation of state are now available. These tables cover the temperature range $5 \times 10^{-3}$ to $1 \times 10^{8}$ $\mathrm{K}$, the density range $10^{-14}$ to $10^{5} \mathrm{~g} / \mathrm{cm}^{3}$, the hydrogen mass fraction $(X)$ range 0.0 to 0.8 , and the metallicity $(Z)$ range 0.0 to 0.04 .

The OPAL and MHD (Hummer and Mihalas, 1988; Däppen, Anderson, and Mihalas, 1987) equations of state are generally in good agreement (Däppen, 1992; Christensen-Dalsgaard and Däppen, 1992). Nevertheless, the small residual differences are important at the current level of accuracy of the solar seismic data. In a recent paper Dziembowski, Pamyatnykh, and Sienkiewicz (1992) used helioseismology to test the MHD equation of state. They found evidence that the MHD approach is inadequate for conditions that exist in the fractional solar radius range $r / R=0.85$ to 0.95 . In this case the OPAL EOS fares somewhat better (Rogers, 1994); whereas Christensen-Dalsgaard (section 3 ) has presented a case that samples a different $r / R$ region, where the MHD equation of state seems to be satisfactory. At higher densities, where the Coulomb coupling is outside the range of validity of the Debye-Hückel theory, discrepancies between MHD and OPAL increase. This is because the OPAL equation of state has systematic corrections for stronger coupling, while MHD does not. These differences could affect the modeling of low mass stars.

9. Helioseismology, solar evolution and other physics (P. Demarque, B. Chaboyer, D.B. Guenther, M.H. Pinsonneault)

The last few years have seen rapid progress in solar interior modeling, and standard solar models (SSM) now predict a p-mode oscillation spectrum which agrees, within the estimated uncertainties in the physical input, with the observed oscillation spectrum of the Sun (Guenther et al., 1992a, 1992b; Guzik \& Cox, 1993). This is the result of a number of improvements in the input physics, most notably the advances in opacities for the solar interior (Rogers \& Iglesias, 1994) and the low temperature regions of the Sun (Kurucz, 1991). The agreement between the observed solar p-mode frequencies and the frequencies predicted by the SSM is not yet perfect, however, and understanding these discrepancies has become a focus of our research.

The importance of diffusion processes, not normally included in the SSM, has been explored and found to be significant (Proffitt \& Michaud, 1991; Bahcall \& Pinsonneault, 1992a,b; Guenther et al., 1993; Guzik \& 
Cox 1993). The diffusion of helium from the surface convection zone into the radiative layers just below modifies the predicted frequencies of those $\mathrm{p}$ - modes that are most sensitive to the physical conditions at the base of the convection zone. Thus including helium diffusion brings the frequencies of p-modes with $l=30$ to 50 into closer agreement with observation (Christensen-Dalsgaard et al., 1993; Guenther et al., 1993; Guzik \& Cox 1993). And the depth of the convection zone of the solar models with diffusion agrees more closely with the depth derived from inversion of the solar p-modes (Christensen-Dalsgaard et al., 1991). Even better agreement was found when the efficiency of diffusion was reduced by a factor of two (see also below). Equally interesting is the result that the improvement brought about by diffusion to the frequencies for modes with $l=30$ to 50 , is at the expense of the calculated frequencies of other p-modes, i.e. modes with $l=50$ to 100 , which do not penetrate to the base of the convection zone (Guenther et al., 1993).

In parallel with our work on diffusion in the solar interior during its past evolution, we have studied the evolution of the Sun as a rotating star in the spirit of Endal \& Sofia $(1978,1981)$. This work has been carried out with a special version of the Yale stellar evolution code (Pinsonneault et al., 1989,1990). The non-linear interaction between rotationally induced mixing and diffusion has been modeled in a self-consistent way during solar evolution by Chaboyer (1993); see also Chaboyer et al. (1995a). A helioseismic study of these models (Chaboyer et al., 1995b) reveals an improvement in the depth of the solar convection zone over purely diffusive models, and also an improvement in the p-mode frequencies for $l=30$ to 50 . Because the rotating models differ little from the SSM (plus some helium diffusion) in structure, they do not resolve the problem mentioned above with the p-mode frequencies in the range $l=50$ to 100 . We conclude that the problem is likely to reside in the opacities near the base of the convection zone, and/or in the details of the very outer layers of our solar models.

The radial distribution of internal angular momentum in the present Sun can be tested directly by observations of the rotational splitting of oscillation modes which penetrate to different depths in the solar interior. Some of the models described in Chaboyer et al. (1995a) are compatible with the inversions of the lowest l-modes observed by IPHIR (Toutain \& Fröhlich 1992) and the IRIS network data (Loudagh et al., 1993), which yield a rotation rate at $0.2 R_{\odot}$ that is higher than the observed solar surface value. However, even our minimal differential rotation model predicts a rotation rate which is incompatible with the nearly flat rotation curve derived from the intermediate $l$ splitting data (Libbrecht et $a l$., 1990). This conclusion is corroborated by the measurement of solar oblateness by Sofia et al. (1994) which constrains the amount of angular momentum in the 
outer solar envelope. Our formulation does not remove angular momentum from the outer parts of the radiative zone with the required efficiency and an important physical process is either missing or inadequately described in our models.

An important consequence of helioseismology is our increased confidence in the validity of the SSM. Further improvements in the nuclear energy generation processes in the Sun and in diffusion coefficients in the solar interior (Bahcall \& Loeb, 1990) have further refined on the SSM (Bahcall \& Pinsonneault, 1992a,b). Helioseismology also provides an excellent opportunity to test non-standard solar models (NSSM). By narrowing down on the range of plausible solar models, helioseismology will enhance our ability to use observations of solar neutrinos to study low energy particle physics.

There has recently been a resurgence of interest in the context of cosmological inflation in the possibility of varying $G$ cosmology and that Newton's constant of gravitation may be a function of time in a Brans-Dicke like manner (Accetta et al., 1990; Krauss \& White, 1992). We have been able to set limits on varying-G during the evolution of the Sun using the observed solar p-mode spectrum (Demarque et al., 1994). This is basically because the depth of the solar convection zone is affected by varying $\mathrm{G}$. This work has been extended to solar g-modes (Guenther et al., 1995), which were found to have even greater sensitivity to varying $\mathrm{G}$. This result is consistent with the greater sensitivity of g-modes to the density distribution near the center of the Sun.

Helioseismology will continue to offer a great opportunity to test the evolution of rotating stars. The physics of the outer layer of the Sun, where current models fail (Guenther, 1994), in particular the superadiabatic layer just below the photosphere, will become amenable to more realistic modeling with the advent of the GONG network and the launching of the SOHO space mission in the near future.

\section{The solar interior and BISON observations (Y. Elsworth)}

The data taken by the Birmingham Solar Oscillation Network (BISON) forms an interesting link between the twin themes of this session: solar and stellar seismology. Like stellar astronomers, we view the Sun as a star without spatial resolution. There are clearly disadvantages in this, in that we lose information about oscillations which vary rapidly across the Sun. But we are not limited to just the simple radial mode which has $l=0$; because of Doppler imaging we see modes with a range of $l$ from 0 to 4 . Unlike stellar astronomers, our star is near to us and the photon flux is high enough that we need not be limited by photon shot noise. We detect the modes by measuring the integrated surface velocity of the Sun. With long inte- 